\title{
A COMMON UNIQUE FIXED POINT THEOREM FOR TWO RANDOM OPERATORS IN HILBERT SPACES
}

\author{
BINAYAK S. CHOUDHURY
}

Received 10 July 2000

\begin{abstract}
We construct a sequence of measurable functions and consider its convergence to the unique common random fixed point of two random operators defined on a nonempty closed subset of a separable Hilbert space. The corresponding result in the nonrandom case is also obtained.
\end{abstract}

2000 Mathematics Subject Classification: 47H10.

1. Introduction. In recent years, the study of random fixed points have attracted much attention, some of the recent literatures in random fixed points may be noted in $[1,2,3,7,8,9]$. In particular, random iteration schemes leading to random fixed points of random operators have been discussed in [3, 4, 5]. In the present paper, we work out a common random fixed point theorem for two random operators defined on a nonempty closed subset of a separable Hilbert space. For the purpose of obtaining the random fixed point of the two random operators we have constructed a sequence of measurable functions and have shown its convergence to the fixed point.

We first review the following concepts which are essentials for our study in this paper.

Throughout this paper, $(\Omega, \Sigma)$ denotes a measurable space, $H$ stands for a separable Hilbert space, and $C$ is a nonempty subset of $H$.

A function $f: \Omega \rightarrow C$ is said to be measurable if $f^{-1}(B \cap C) \in \Sigma$ for every Borel subset $B$ of $H$. A function $f: \Omega \times C \rightarrow C$ is said to be a random operator, if $F(\cdot, x): \Omega \rightarrow C$ is measurable for every $x \in C$. A measurable function $g: \Omega \rightarrow C$ is said to be a random fixed point of the random operator $F: \Omega \times C \rightarrow C$, if $F(t, g(t))=g(t)$ for all $t \in \Omega$. A random operator $F: \Omega \times C \rightarrow C$ is said to be continuous if for fixed $t \in \Omega, F(t, \cdot): C \rightarrow C$ is continuous.

Condition 1.1. Two mappings $S, T: C \rightarrow C$, where $C$ is a nonempty subset of a Hilbert space $H$, is said to satisfy Condition 1.1 if

$$
\begin{aligned}
\|S x-T y\|^{2} \leq & a\|x-y\|^{2}+b\left(\|x-S x\|^{2}+\|y-T y\|^{2}\right) \\
& +\frac{c}{2}\left(\|x-T y\|^{2}+\|y-S x\|^{2}\right),
\end{aligned}
$$

where

$$
0<a+2 b+2 c<1, \quad a, b, c>0 .
$$


It is well known that in a Hilbert space the parallelogram law is satisfied, that is,

$$
\forall x, y \in C, \quad\|x+y\|^{2}+\|x-y\|^{2}=2\|x\|^{2}+2\|y\|^{2} .
$$

We construct a sequence of functions $\left\{g_{n}\right\}$ as

$$
g_{0}: \Omega \longrightarrow C
$$

is arbitrary measurable function. For $t \in \Omega$ and $n=0,1,2, \ldots$,

$$
g_{2 n+1}(t)=S\left(t, g_{2 n}(t)\right), \quad g_{2 n+2}(t)=T\left(t, g_{2 n+1}(t)\right) .
$$

2. Main results. In this section, we prove a common unique fixed point theorem for two random operators in Hilbert spaces.

THEOREM 2.1. Let $C$ be a nonempty closed subset of a separable Hilbert space $H$. Let $S$ and $T$ be two continuous random operators defined on $C$ such that for $t \in \Omega$, $S(t, \cdot), T(t, \cdot): C \rightarrow C$ satisfy Condition 1.1. Then the sequence $\left\{g_{n}\right\}$ obtained in (1.4) and (1.5) converges to the unique common random fixed point of $S$ and $T$.

Proof. For fixed $t \in \Omega, n=1,2 \ldots$,

$$
\begin{aligned}
\left\|g_{2 n+1}(t)-g_{2 n}(t)\right\|^{2}= & \left\|S\left(t, g_{2 n}(t)\right)-T\left(t, g_{2 n-1}(t)\right)\right\|^{2} \\
\leq & a\left\|g_{2 n}(t)-g_{2 n-1}(t)\right\|^{2} \\
& +b\left(\left\|g_{2 n}(t)-S\left(t, g_{2 n}(t)\right)\right\|^{2}+\left\|g_{2 n-1}(t)-T\left(t, g_{2 n-1}(t)\right)\right\|^{2}\right) \\
& +\frac{c}{2}\left(\left\|g_{2 n}(t)-T\left(t, g_{2 n-1}(t)\right)\right\|^{2}+\left\|g_{2 n-1}(t)-S\left(t, g_{2 n}(t)\right)\right\|^{2}\right) \\
= & a\left\|g_{2 n-1}(t)-g_{2 n}(t)\right\|^{2} \\
& +b\left(\left\|g_{2 n}(t)-g_{2 n+1}(t)\right\|^{2}+\left\|g_{2 n-1}(t)-g_{2 n}(t)\right\|^{2}\right) \\
& +\frac{c}{2}\left(\left\|g_{2 n}(t)-g_{2 n}(t)\right\|^{2}+\left\|g_{2 n-1}(t)-g_{2 n+1}(t)\right\|^{2}\right) \\
= & a\left\|g_{2 n}(t)-g_{2 n-1}(t)\right\|^{2} \\
& +b\left(\left\|g_{2 n}(t)-g_{2 n+1}(t)\right\|^{2}+\left\|g_{2 n-1}(t)-g_{2 n}(t)\right\|^{2}\right) \\
& +\frac{c}{2}\left\|\left(g_{2 n-1}(t)-g_{2 n}(t)\right)+\left(g_{2 n}(t)-g_{2 n+1}(t)\right)\right\|^{2} \\
= & a\left\|g_{2 n}(t)-g_{2 n-1}(t)\right\|^{2} \\
& +b\left(\left\|g_{2 n}(t)-g_{2 n+1}(t)\right\|^{2}+\left\|g_{2 n-1}(t)-g_{2 n}(t)\right\|^{2}\right) \\
& +c\left(\left\|g_{2 n-1}(t)-g_{2 n}(t)\right\|^{2}+\left\|g_{2 n}(t)-g_{2 n+1}(t)\right\|^{2}\right) \\
& -\frac{c}{2}\left(\left\|\left(g_{2 n-1}(t)-g_{2 n}(t)\right)-\left(g_{2 n}(t)-g_{2 n+1}(t)\right)\right\|^{2}\right)
\end{aligned}
$$

(by parallelogram law (1.3))

$$
\begin{aligned}
\leq & (a+b+c)\left\|g_{2 n}(t)-g_{2 n-1}(t)\right\|^{2} \\
& +(b+c)\left\|g_{2 n}(t)-g_{2 n+1}(t)\right\|^{2} .
\end{aligned}
$$


Therefore,

$$
\left\|g_{2 n+1}(t)-g_{2 n}(t)\right\|^{2} \leq \frac{a+b+c}{1-b-c}\left\|g_{2 n}(t)-g_{2 n-1}(t)\right\|^{2} .
$$

For $t \in \Omega, n=1,2,3, \ldots$,

$$
\begin{aligned}
& \left\|g_{2 n}(t)-g_{2 n-1}(t)\right\|^{2}=\left\|T\left(t, g_{2 n-1}(t)\right)-S\left(t, g_{2 n-2}(t)\right)\right\|^{2} \\
& =a\left\|g_{2 n-2}(t)-g_{2 n-1}(t)\right\|^{2} \\
& +b\left(\left\|g_{2 n-1}(t)-T\left(t, g_{2 n-1}(t)\right)\right\|^{2}\right. \\
& \left.+\left\|g_{2 n-2}(t)-S\left(t, g_{2 n-2}(t)\right)\right\|^{2}\right) \\
& +\frac{c}{2}\left(\left\|g_{2 n-1}(t)-S\left(t, g_{2 n-2}(t)\right)\right\|^{2}+\left\|g_{2 n-2}(t)-T\left(t, g_{2 n-1}(t)\right)\right\|^{2}\right) \\
& \text { (by }(1.1) \text { ) } \\
& =a\left\|g_{2 n-2}(t)-g_{2 n-1}(t)\right\|^{2} \\
& +b\left(\left\|g_{2 n-1}(t)-g_{2 n}(t)\right\|^{2}+\left\|g_{2 n-2}(t)-g_{2 n-1}(t)\right\|^{2}\right) \\
& +\frac{c}{2}\left(\left\|g_{2 n-1}(t)-g_{2 n-1}(t)\right\|^{2}+\left\|g_{2 n-2}(t)-g_{2 n}(t)\right\|^{2}\right) \\
& =a\left\|g_{2 n-1}(t)-g_{2 n-2}(t)\right\|^{2} \\
& +b\left(\left\|g_{2 n-1}(t)-g_{2 n}(t)\right\|^{2}+\left\|g_{2 n-2}(t)-g_{2 n-1}(t)\right\|^{2}\right) \\
& +\frac{c}{2}\left\|\left(g_{2 n-2}(t)-g_{2 n-1}(t)\right)+\left(g_{2 n-1}(t)-g_{2 n}(t)\right)\right\|^{2} \\
& \leq a\left\|g_{2 n-1}(t)-g_{2 n-2}(t)\right\|^{2} \\
& +b\left(\left\|g_{2 n-1}(t)-g_{2 n}(t)\right\|^{2}+\left\|g_{2 n-2}(t)-g_{2 n-1}(t)\right\|^{2}\right) \\
& +c\left(\left\|g_{2 n-2}(t)-g_{2 n-1}(t)\right\|^{2}+\left\|g_{2 n-1}(t)-g_{2 n}(t)\right\|^{2}\right) \\
& -\frac{c}{2}\left\|\left(g_{2 n-2}(t)-g_{2 n-1}(t)\right)-\left(g_{2 n-1}(t)-g_{2 n}(t)\right)\right\|^{2} \\
& \text { (by parallelogram law) } \\
& \leq(a+b+c)\left\|g_{2 n-1}(t)-g_{2 n-2}(t)\right\|^{2} \\
& +(b+c)\left\|g_{2 n-1}(t)-g_{2 n}(t)\right\|^{2} .
\end{aligned}
$$

Therefore, for all $t \in \Omega, n=1,2, \ldots$,

$$
\left\|g_{2 n}(t)-g_{2 n-1}(t)\right\|^{2} \leq\left(\frac{a+b+c}{1-b-c}\right)\left\|g_{2 n-1}(t)-g_{2 n-2}(t)\right\|^{2}
$$


Equations (2.2) and (2.4) jointly imply that for all $t \in \Omega, n=1,2,3, \ldots$,

$$
\left\|g_{n}(t)-g_{n+1}(t)\right\|^{2} \leq\left(\frac{a+b+c}{1-b-c}\right)\left\|g_{n-1}(t)-g_{n}(t)\right\|^{2}
$$

Again from (1.2) it follows that

$$
0<\left(\frac{a+b+c}{1-b-c}\right)<1
$$

From (2.5) and (2.6) it follows that for $t \in \Omega,\left\{g_{n}(t)\right\}$ is a Cauchy sequence and hence is convergent in the Hilbert space $H$.

For $t \in \Omega$, let

$$
\left\{g_{n}(t)\right\} \rightarrow g(t) \quad \text { as } n \longrightarrow \infty
$$

Since $C$ is closed, $g$ is a function from $C$ to $C$.

For $t \in \Omega$,

$$
\begin{aligned}
&\|g(t)-S(t, g(t))\|^{2}=\left\|\left(g(t)-g_{2 n}(t)\right)+\left(g_{2 n}(t)-S(t, g(t))\right)\right\|^{2} \\
& \leq 2\left\|g(t)-g_{2 n}(t)\right\|^{2}+2\left\|g_{2 n}(t)-S(t, g(t))\right\|^{2} \\
& \quad(\text { by parallelogram law }) \\
&= 2\left\|g(t)-g_{2 n}(t)\right\|^{2}+2\left\|T\left(t, g_{2 n-1}(t)\right)-S(t, g(t))\right\|^{2} \\
&= 2\left\|g(t)-g_{2 n}(t)\right\|^{2}+2 a\left\|g_{2 n-1}(t)-g(t)\right\|^{2} \\
&+2 b\left(\left\|g_{2 n-1}(t)-T\left(t, g_{2 n-1}(t)\right)\right\|^{2}+\|g(t)-S(t, g(t))\|^{2}\right) \\
&+c\left\|g_{2 n-1}(t)-S(t, g(t))\right\|^{2}+\left\|g(t)-T t, g_{2 n-1}(t)\right\|^{2} \\
& \\
&= 2\left\|g(t)-g_{2 n}(t)\right\|^{2}+2 a\left\|g_{2 n-1}(t)-g(t)\right\|^{2} \\
&+2 b\left(\left\|g_{2 n-1}(t)-g_{2 n}(t)\right\|^{2}+\|g(t)-S(t, g(t))\|^{2}\right) \\
&+c\left(\left\|g_{2 n-1}(t) S(t, g(t))\right\|^{2}+\left\|g(t)-g_{2 n}(t)\right\|^{2}\right) .
\end{aligned}
$$

Making $n \rightarrow \infty$ in the above inequality we have by virtue of (2.7), for all $t \in \Omega$,

$$
\|g(t)-S t, g(t)\|^{2} \leq(2 b+c)\|g(t)-S(t, g(t))\|^{2} .
$$

Since $0<2 b+c<1$ (by (1.2)), we have for all $t \in \Omega$,

$$
S(t, g(t))=g(t)
$$

In an exactly similar way we can prove that for all $t \in \Omega$,

$$
T(t, g(t))=g(t)
$$


Again, if $A: \Omega \times C \rightarrow C$ is a continuous random operator on a nonempty subset $C$ of a separable Hilbert space $H$, then for any measurable function $f: \Omega \rightarrow C$, the function $h(t)=A(t, f(t))$ is also measurable [6].

It follows from the construction of $\left\{g_{n}\right\}((1.4)$ and (1.5)) and the above considerations that $\left\{g_{n}\right\}$ is a sequence of measurable functions. From (2.7) it follows that $g$ is also a measurable function. This fact along with (2.10) and (2.11) shows that $g: \Omega \rightarrow C$ is a common random fixed point of $S$ and $T$.

Next we prove the uniqueness. Let $h: \Omega \rightarrow C$ be another random fixed point common to $S$ and $T$, that is, for $t \in \Omega$,

$$
S(t, h(t))=h(t), \quad T(t, h(t))=h(t) .
$$

Then for $t \in \Omega$,

$$
\begin{aligned}
\|g(t)-h(t)\|^{2}= & \|S(t, g(t))-T(t, h(t))\|^{2} \\
\leq & a\|g(t)-h(t)\|^{2}+b\left(\|g(t)-S(t, g(t))\|^{2}+\|h(t)-T(t, h(t))\|^{2}\right) \\
& +\frac{c}{2}\left(\|g(t)-T(t, h(t))\|^{2}+\|h(t)-S(t, g(t))\|^{2}\right) \\
= & (a+c)\|g(t)-h(t)\|^{2} \quad(\text { by }(2.12)) .
\end{aligned}
$$

But $0<a+c<1$ (by (1.2)). This shows that $g(t)=h(t)$ for all $t \in \Omega$. This completes the proof of the theorem.

COROLlary 2.2. Let $S, T: C \rightarrow C$, where $C$ is a nonempty closed subset of a Hilbert space $H$, be such that inequality (1.1) is satisfied along with (1.2). Then the sequence obtained by starting with an arbitrary element

$$
\begin{gathered}
x_{0} \in C, \\
x_{2 n+1}=S x_{2 n}, \quad n=0,1,2, \ldots, \\
x_{2 n+2}=T x_{2 n+1}, \quad n=0,1,2, \ldots,
\end{gathered}
$$

converges to a unique common fixed point of $S$ and $T$.

The proof of the corollary is immediate by assuming $\Omega$ to be a singleton set.

REMARK 2.3. It is necessary to assume $H$ to be separable in the corollary.

ACKNOWLEDGMENT. This work was supported by a grant from Bengal Engineering College (a Deemed University). The support is gratefully acknowledged.

\section{REFERENCES}

[1] I. Beg and N. Shahzad, Random fixed points of random multivalued operators on Polish spaces, Nonlinear Anal. 20 (1993), no. 7, 835-847.

[2] __ Random approximations and random fixed point theorems, J. Appl. Math. Stochastic Anal. 7 (1994), no. 2, 145-150.

[3] B. S. Choudhury, Convergence of a random iteration scheme to a random fixed point, J. Appl. Math. Stochastic Anal. 8 (1995), no. 2, 139-142. 
[4] B. S. Choudhury and M. Ray, Convergence of an iteration leading to a solution of a random operator equation, J. Appl. Math. Stochastic Anal. 12 (1999), no. 2, 161-168.

[5] B. S. Choudhury and A. Upadhyay, An iteration leading to random solutions and fixed points of operators, Soochow J. Math. 25 (1999), no. 4, 395-400.

[6] C. J. Himmelberg, Measurable relations, Fund. Math. 87 (1975), 53-72.

[7] N. S. Papageorgiou, Random fixed point theorems for measurable multifunctions in Banach spaces, Proc. Amer. Math. Soc. 97 (1986), no. 3, 507-514.

[8] V. M. Sehgal and C. Waters, Some random fixed point theorems for condensing operators, Proc. Amer. Math. Soc. 90 (1984), no. 3, 425-429.

[9] H. K. Xu, Some random fixed point theorems for condensing and nonexpansive operators, Proc. Amer. Math. Soc. 110 (1990), no. 2, 395-400.

Binayak S. Choudhury: Department of Mathematics, Bengal Engineering College (DEEMED UNIVERSITY), HOWRAH 711103, WEST BENGAL, INDIA

E-mail address: bsc@math . becs . ac. in 


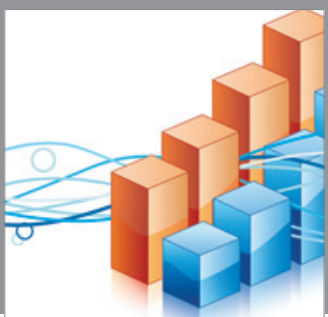

Advances in

Operations Research

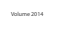

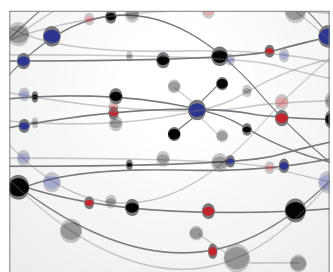

\section{The Scientific} World Journal
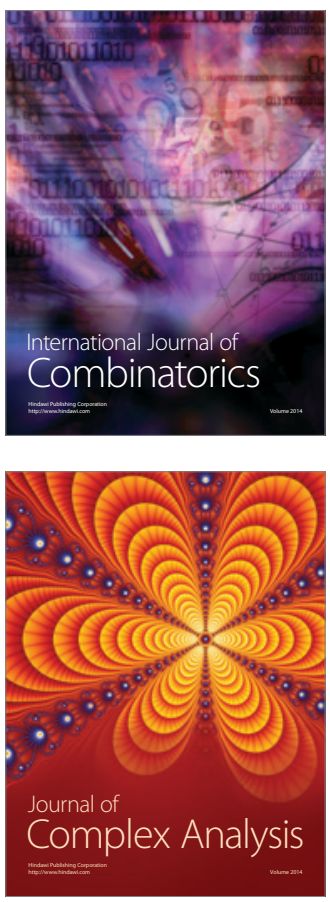

International Journal of

Mathematics and

Mathematical

Sciences
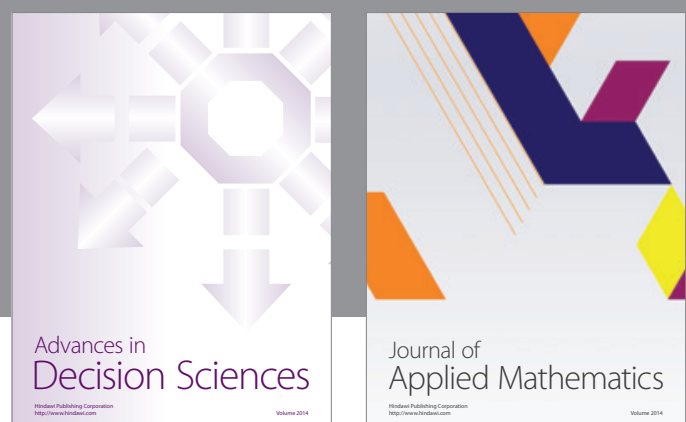

Journal of

Applied Mathematics
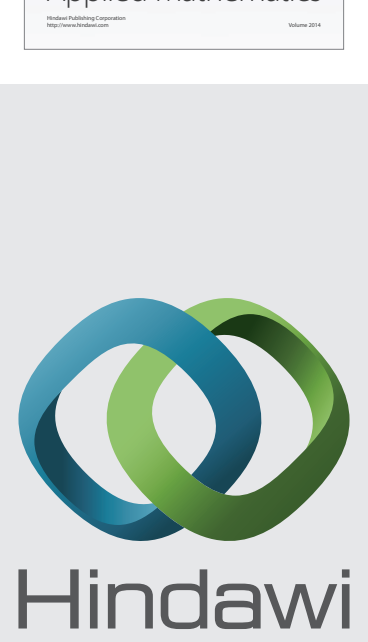

Submit your manuscripts at http://www.hindawi.com
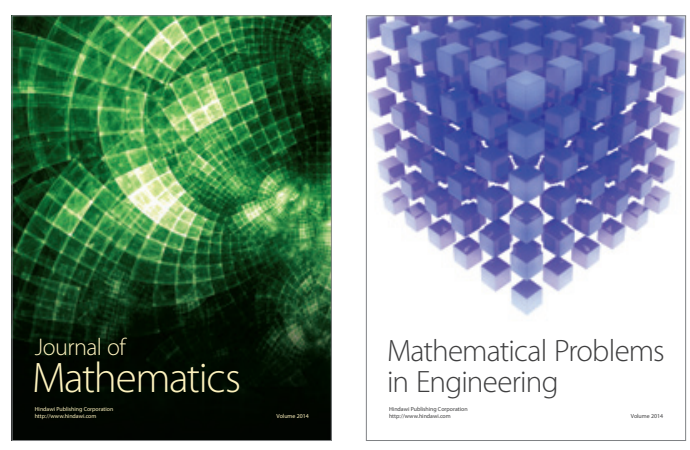

Mathematical Problems in Engineering
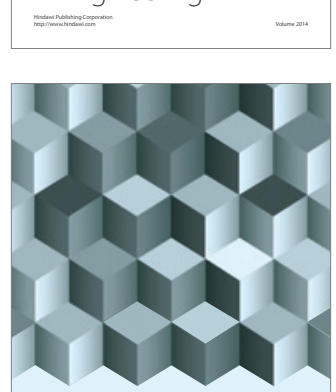

Journal of

Function Spaces
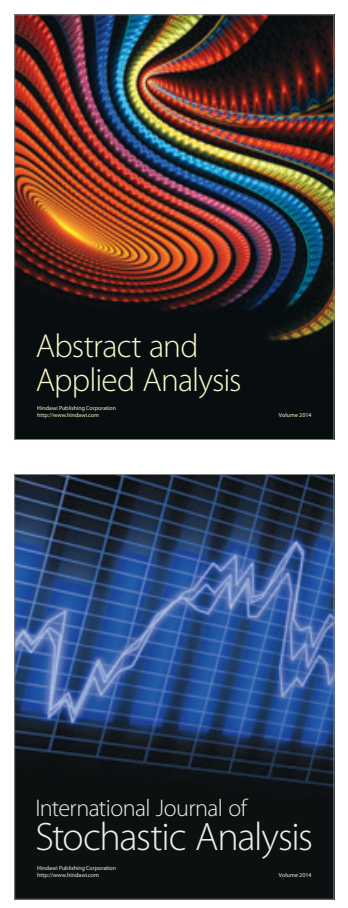

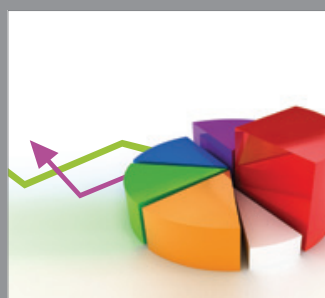

ournal of

Probability and Statistics

Promensencen
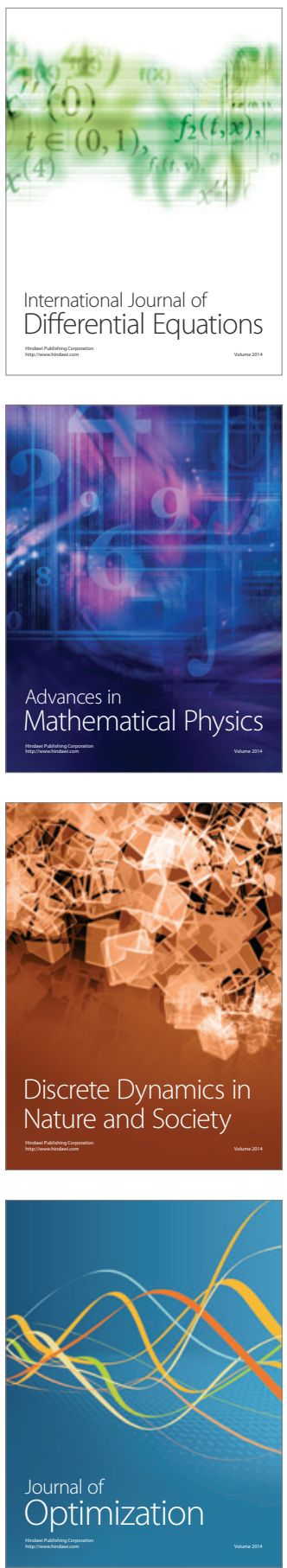